\title{
EvoS: A Prescription for What Ails Pre-Medical Education?
}

\author{
Jennifer Turner Waldo • Stacy A. Greagor
}

Published online: 25 January 2011

(C) Springer Science+Business Media, LLC 2011

\begin{abstract}
Pre-medical students are certainly a widely varied group, with different motivations and experiences, different skills sets and interests. However, they often tend to approach their undergraduate education as a necessary evil that they must endure in order to achieve their ultimate goals. This article summarizes recent literature addressing some of the questions that have been raised regarding premedical education programs. Are students prepared for the intellectual, emotional, and even physical challenges of medical training? What deficiencies are commonly seen in entering medical students? What are students' perceptions of how well their pre-medical studies helped them? Many of these studies have resulted in a call for more science training, while some have advocated for less, but with an enhanced focus on humanistic studies. We supply a brief outline of our Evolutionary Studies (EvoS) program and reflect upon how participation in this program can enhance pre-medical students' education. Importantly, we argue that EvoS can expand students' depth of understanding of science, as well as nurture their ability to think about the needs of their patients and the context of their medical practice.
\end{abstract}

Keywords Evolutionary studies $($ EvoS $) \cdot$ Pre-medical education · Evolution

A student showed us a neat trick. He wrote a single word on a piece of paper and asked some of his fellow students to simply read it out loud. The students were all of the premedical persuasion, doing well in their coursework and

J. T. Waldo $(\bowtie) \cdot$ S. A. Greagor

Biology Department, State University of New York at New Paltz, 1 Hawk Drive,

New Paltz, NY 12561, USA

e-mail: waldoj@newpaltz.edu engaged in their studies. The word was UNIONIZED. Surprisingly, the majority of the students said "un-ion-ized," rather than "union-ized."

For those that are not sure, "un-ion-ized" is not a wordit has no meaning. But these students picked out the "ion" part of the word as being most meaningful, rather than seeing a relatively commonly used word. The term "ion" is very important in chemistry, but it generally is not part of "normal" conversations. Why did they do this? What does it mean about them and the education they are receiving? How does this relate to their future careers in medicine?

\section{What is Wrong with Pre-Medical Education?}

When approached by prospective students about the quality of the pre-medical major at our college, one must respond by saying that we do not have a pre-med major. In fact, most colleges and universities do not, and this is particularly true for colleges that focus on a liberal arts education, like our own. Instead, pre-med is a "state of mind." It is a selfidentification and it really is not a valid designation until one is actually enrolled in medical school. Somebody that takes pre-med courses but does not gain entrance into medical school is not truly pre-med.

Pre-med students are goal-oriented by definition. There are particular lists of classes, experiences and tests that these students must complete to achieve their goal. There are as many motivators that result in the production of a pre-med mentality as there are students that identify as such. But, in our experience, there are usually some aspects of family or cultural respect for the career, glamorization of doctors in entertainment, a true desire to help others and perceived financial incentives that contribute to the decision to pursue medicine as a career. 
In the US, thousands of students each year start their journey to becoming doctors by enrolling in a Bachelor's degree program. For many of these students, this journey ends in frustration as they are unable to master the material presented in introductory science classes (Barr et al. 2008). For those that persist, many may be unable to see how what they learn in college is applicable to their ultimate goals in medicine. Many strive to achieve the highest grades possible, rather than truly understand the material, as it is clearly stated that high grades will get them into the medical school of their choice. This quest for a perfect GPA becomes the struggle of their college education, and because of this, they may miss out on an important part of their development. In addition, the quest for the highest grades is often perceived to be a zero-sum pursuit, so competition rather than collaboration is often the norm. Thus, the pre-med years are viewed more as a "tourney than a journey" (Gross et al. 2008).

If you would like an insight into the world of many premeds, visit the website www.studentdoctor.net and click on the pre-med forums tab. Here, you can find over four million posts by undergraduates seeking assistance in course selection, soliciting letters of recommendation, securing beneficial extracurricular experiences, manipulating their GPA, preparing for taking (or re-taking) the MCAT and psyching themselves up for interviews. There is an entire section entitled "what are my chances," in which pre-meds critique each other's qualifications and predict how likely an applicant is to gain medical school admission. Rarely, if at all, does one find posts about the content of the coursework and how it will affect their ability to practice medicine. Virtually all of the posts see the entire process only in terms of the immediate outcome-admission to (hopefully a good) medical school.

While there are many routes through a pre-medical curriculum, the basic requirements for medical school admission are: a satisfactory score on the MCAT, and a year of general biology, chemistry, physics, and organic chemistry (www.aamc.org/students/applying/requirements/). Clearly, these disciplines have aspects that relate directly to the practice of medicine. Humans are, after all, biological organisms subject to the laws of the physical universe, most drugs are chemicals and most medical equipment relies upon advances in physics. Still, our personal experience is that a cell biology teacher may have to frequently defend the reasons that a person wanting to be a brain surgeon needs to know about glycolysis or the function of the Golgi complex. Of course, there are good answers to these queries. But wouldn't it be better if the students could figure this out on their own? And shouldn't they be able to if these requirements are important to their future careers?

Oftentimes, physics and organic chemistry are the subjects that pre-meds are least able to relate to their career goals (Barr et al. 2008). Paradoxically, we have observed that pre-meds tend to afford them an inordinate amount of their study time and efforts. For example, in our school, pre-meds are often co-enrolled in genetics and organic chemistry and the demands of organic chemistry are frequently cited as an excuse for lack of time spent focusing on genetics. Thus, the students who respond "un-ion-ized" to the prompt of unionized are doing so likely because they can see only the "trees" of organic chemistry and do not appreciate how the disparate facts of the pre-medical curriculum fit into a coherent framework.

Perhaps most surprising is not that both students and faculty are unsatisfied with the current state of pre-medical education, but that there is really a serious dearth of data analyzing the effectiveness of this system (Barr et al. 2008; Gross et al. 2008). Like many aspects of education, it has persisted probably because it is a truly daunting task to thoughtfully address the issues and come up with a plan to improve it.

But does any of this angst about pre-medical education really mean that we, as a country or culture, are missing out on something because of these shortcomings? Many, including the current president of the American Association of Medical Colleges, argue that yes, we are (Kirch 2010). Study after study has shown that while we are increasing the social and racial diversity of practicing doctors in the US, we are far from an era where the physician workforce is truly reflective of the population (Ward 2010). The most glaring example of this is the lack of Hispanic doctors (AAMC 2010). This is not just a failure in a theoretical utopian quest for equity, it is a real problem that inhibits an increasing percentage of our population from achieving parity in health outcomes.

In addition, the pool of physicians selected for by the current pre-medical and medical education systems may be enriched for individuals that encounter difficulties dealing with some of the stressful aspects of their chosen careers. For example, physicians have higher suicide rates than the general population (AMA 1987). These physicians are often reticent to seek psychological help, as there are potential career-ending consequences to doing so (Center et al. 2003). In addition, while health care is always going to be prone to "human error," one could ask questions about the relationship between how we are selecting physicians and the high rate of medical errors reported (West et al. 2006).

Finally, there is actually a physician shortage in this country (O'Reilly 2010). Despite the seemingly endless numbers of pre-med students, the fact is that we are not producing enough doctors - especially general care practitioners - to keep up with demand. In some lucrative specialties, however, there are more doctors than required (Harris 2010). One could argue that the pre-medical education system has at least 
some responsibility for this imbalance by selecting students that do not choose primary care practices for their career.

\section{What Can Be Done About Pre-Med Education?}

A little over a hundred years ago, Flexner proposed the premedical and medical curriculum that has been remarkably persistent to the present day (Flexner 1910). This was a standardization of the medical education system that was necessitated by widely varying types of medical practice throughout the country and a desire to create a minimum set of standards for physicians. In this report, the requirement for education beyond high school was instituted and a firm basis in basic sciences was proposed. The fact that we are still educating physicians within this general framework is a testimony to its success. However, there has been an increasing call for revisiting the recommendations of Flexner and coming up with a system that addresses modern issues in both medical and pre-medical education (Beck 2004).

As might be guessed, there are dramatically different suggestions out there about how to fix the perceived problems in pre-med education. Basically, they fall into five distinct groups: eliminate all requirements; emphasize the basic sciences more; emphasize the basic sciences less; eliminate specific course requirements, but have more flexibly defined competences; and, finally, include more emphasis on evolutionary biology.

One of the most radical proposals for change came from respected scholar-poet-physician Lewis Thomas (1978). Dr. Thomas advocated for eliminating even the idea of premedical education, favoring instead a truly liberal arts experience. In fact, according to Dr. Thomas, students should study Greek and focus on the classics. This would put the onus on the medical school curriculum to impart all of the science knowledge future physicians need.

If Dr. Thomas' proposal is too radical, there is a more moderate approach that advocates for retaining some of the basic science requirements (general biology and chemistry) while eliminating others (organic chemistry, physics, and the MCAT) (Emanuel 2006; Dalen and Alpert 2009). This model has been embraced by a program administered by the Mt. Sinai School of Medicine for the past 23 years. This program requires students to major in the social sciences or humanities and provides some additional academic support prior to the beginning of medical school classes. This approach was recently assessed (Muller and Kase 2010) with the finding that students in the program had similar outcomes to those with a traditional pre-medical background. One interesting finding was that the non-traditional pre-meds tended to choose more general practice specialties than their traditional counterparts.
On the opposite end of the spectrum, some advocate for enhancing the depth and rigor of training in the basic sciences (Dienstag 2008). Those making this argument believe that this preparation would enable medical students to explore, in more depth, the sciences of particular relevance to medicine. In particular, Dienstag argues that the era of personal, genetics-based medicine (PMC 2009) requires that biochemistry, genetics, and cell/molecular biology be viewed as basic sciences that must be mastered (in depth) during the pre-medical years and built upon in medical school. However, this would need to come at the expense of coursework outside of the sciences and may be problematic to implement at a consistent level across so many different types of colleges and programs (Austin 2008).

Perhaps the most "reasonable" suggestion is that proposed by a group from the Association of American Medical Colleges and Howard Hughes Medical Institute (AAMC-HHMI). Representatives from a variety of interests in pre-medical and medical education convened to re-imagine requirements for admission to and within medical school (AAMC-HHMI 2010). Because this group represents the medical colleges themselves, their proposal can be regarded as an expression of the direction these institutions see moving in the future. The group determined that rather than prepare a list of specific course requirements, they would provide a list of competencies and learning outcomes to be attained. While a lot of thought has gone into this, it is a bit unclear as to how this can be implemented and its success assessed (dePaula 2009).

One of the competencies for pre-medical education that was reported by the AAMC-HHMI group was that students master proficiency in evolutionary biology prior to enrolling in medical school. This expression of the utility of evolutionary biology to medical education resulted in convening a group of scientists and physicians to advocate for the enhancement of this recommendation (Nesse et al. 2010). We agree with these authors that evolutionary biology provides an important framework upon which medical education should be based. The field of evolutionary medicine is an increasingly relevant field of study in medicine (Wilson 2007) that endeavors to see human bodies not as perfectly designed machines, but as the product of imperfect and on-going selection pressures. Thus, disease and illness should be studied and treated with an eye towards the evolutionary histories that cause them to result.

\section{What Is EvoS, and How Can It Help?}

Evolutionary Studies (EvoS) is a recent and growing interdisciplinary trend in academia. Started at Binghamton 
University by David Sloan Wilson and others, similar programs can now be found at increasing numbers of colleges in the US and beyond. Each program is different and based on maximizing strengths and minimizing administrative pitfalls at each institution. But, generally, each result in a minor (or equivalent) upon completion and the requirements include introductory courses in evolutionary theory, an experience into the breadth of evolutionary sciences and participation in a seminar-style course that allows students direct interaction with experts from an incredible variety of academic fields that are united in using evolutionary theory as a framework upon which to build their scholarship (Wilson et al. 2009).

Rather than see evolution as strictly in the domain of biology, EvoS places particular emphasis on how evolution has shaped the human experience and explores the application of evolutionary theory to the natural and social sciences, humanities and the arts.

The campuses that have had success in developing EvoS programs have formed a consortium to share resources that has been supported by a National Science Foundation Course, Curriculum and Laboratory Improvement grant (\#0817337). As a result of this support, starter-grants for new programs have been awarded to several campuses, new courses have been developed, student-faculty research projects have been funded and a freely accessible website has been developed (http://www.evostudies.org). The website contains a repository of educational materials that can be shared across campuses, access to assessment tools and a journal which publishes peer-reviewed articles relating to evolutionary studies and education. In addition, the journal solicits and publishes papers from undergraduates that are reviewed by a strictly undergraduate-staffed review board.

While EvoS programs were certainly not developed with the specific intent of addressing issues in pre-medical education, it is increasingly clear that they may be able to provide a valuable role in the efforts to improve the premedical experience. Important in this is the interdisciplinary nature of EvoS. This approach is heralded by the AAMCHHMI study (AAMC-HHMI 2010), which states:

Organizing educational programs according to departmental priorities is a long-standing tradition in both undergraduate and professional education, but some institutions have begun to develop their educational program through an integrated, nondepartmental approach, and it is this approach the committee supports in the report.

As described above, the field of evolutionary medicine is becoming a transformative factor in medical practice (Nesse 2008). How much more powerful could it become if premed students begin evolutionary thinking (Wilson 2007) early in their training? In order to truly make progress in some of the big health issues facing us today-obesity, aging, cancer, infectious agents - evolution and comparative biology provide critical insights that must be considered. For example, understanding the way that particular pathogenic viruses or bacteria spread can shed light on the severity of the symptoms that evolved to be associated with the infection. If an insect vector can feed on immobile or incapacitated victims and then spread the disease to other individuals - as in the case of malaria - the symptoms of infection are often severely debilitating. However, if humanto-human contact is required for transmission - as with the common cold virus - the symptoms result in the infected individuals being able to go about most of their daily activities (Nesse 2008). Appreciating this can help physicians to develop appropriate treatment and containment protocols for different infectious agents.

While it is difficult to imagine that pre-medical training will radically change in the near future, it is easy to envision the concept of an acceptable pre-med education will broaden and trend towards encompassing more of the competencies outlined in the AAMC-HHMI report. Therefore, there will likely continue to be pre-meds that choose academic majors in the basic sciences and increasing numbers that obtain training in the basic sciences while pursuing majors in the humanities and social sciences. We believe that EvoS programs provide a unifying framework for all of these students, while providing particular benefits to each group.

For science major pre-meds, EvoS can provide a specific focus on humans and human health. A biology major curriculum is not designed to be all about humans. Ideally, it should be designed to expose its students to the tools needed to appreciate and investigate the wide diversity of life forms on earth (Labov et al. 2010). Clearly, this is a big enough task on its own, without the added burden of providing pre-meds the touchstone with human-specific information that they often crave, and even, need. While pre-med biology majors may receive adequate training in basic evolutionary principles, taking classes to fulfill their EvoS minor requirements and participating in the seminar series will allow them to explore how these principles are specifically applied to the studies of humans.

Perhaps the specific EvoS courses that could prove to be highly beneficial to pre-meds would be those that are taught within the disciplines of psychology and sociology. Specifically, courses such as evolutionary psychology, social psychology, and the psychology of infancy and childhood could help pre-meds to understand people at a different, more comprehensive level than simply the sum of their biological functions. The ultimate goal of completing medical school is to be able to provide care to another human being. Understanding how humans behave, with an eye towards evolutionary histories of such behaviors may 
help physicians treat their patients more humanely, rather than if they were scientific experiments. Pre-med students could also benefit from more abstract EvoS courses that focus on language and the arts. These courses may help to facilitate development in critical thinking, creativity, and reasoning. These skills may help provide physicians with the ability to "think outside of the box" when it comes to diagnosing or treating particularly troubling cases.

In addition, participation in EvoS will likely improve these students' ability to communicate scientific information to a less science literate population. This skill is something that pre-meds often struggle with and is critical to their success in medicine, especially if they choose a general practice career. Improvement in this area will come primarily from two experiences. First, having repeated exposure to seminars from many disciplines models effective (and, at times, ineffective) communication strategies. Second, participation in nonscience based courses often includes more class time spent in discussions, and the science majors may even be relied upon as science experts in this setting.

For those not majoring in basic sciences, EvoS can provide an appreciation for the larger context of life on earth (beyond humans) than is often considered in nonscience courses. Because of this, it provides a logical framework for the basic sciences that is likely to enhance student success. An understanding of evolution and the ability to broadly apply it may help in ameliorating the need for strict memorization and favor a deeper understanding of the material. An often-quoted phrase "nothing in biology makes sense except in the light of evolution" (Dobzhansky 1964), highlights the central relevance of evolution to informing studies in biology. In particular, for pre-med students that do not explore biology in depth by completing a biology major, this perspective may be critical in their ability to synthesize the information they are learning.

The ability to meet other EvoS pre-meds through a shared course and attending the seminar series creates a community of like-minded students that can provide support to each other throughout their studies. This can be particularly important for those students that are taking science courses at times and in a sequence different than that of science majors, who oftentimes follow a quite rigid sequence of classes.

In short, EvoS programs can serve as a home base for pre-meds of all majors. This is likely to help them succeed as pre-meds, as medical students, and in their careers as physicians.

One of the consequences of recommending EvoS for pre-med students, whether they major in a science or not, is that the competitive nature of pre-med students may alter the dynamic of the EvoS program. Students who are not looking to apply to medical school may feel a bit intimidated by pre-meds and turned-off by their single- minded focus. Despite this, we believe that both groups of students can ultimately benefit from each other's presence. Pre-meds will be able to apply their scientific knowledge in EvoS courses, which will offer non-medically minded students new perspectives. In addition, these students will similarly benefit the pre-meds by modeling non-scientific ways of thinking. Finally, we predict that the population of pre-meds with an interest in joining EvoS may be selfselected for students that are more likely to fit into the convivial and cooperative nature of EvoS programs (Wilson et al. 2009).

One of the most attractive aspects of this approach to revitalizing pre-medical education is that it does not involve a whole-scale restructuring of entrenched academic constructs. EvoS is built upon and from components already in existence at all college campuses. In addition, EvoS does not serve only pre-meds; it provides benefits to all students and faculty that participate. A straight-forward assessment of the utility of EvoS in addressing the issues we have outlined could come from following the career trajectories of two groups of matched pre-med students that either participate in an EvoS program, or decline participation. This study should look at success in the pre-medical years and during medical school, as well as examine specialization choices and career satisfaction. We encourage campuses looking to bolster their pre-med programs to consider doing so in the context of EvoS.

Acknowledgment This work was supported by an NSF Course Curriculum and Laboratory Improvement grant \#0817337.

\section{References}

AAMC. America needs a more diverse physician workforce. Retrieved November 15, 2010 from https://www.aamc.org/download/45694/ data/diversity.pdf. 2010.

AAMC-HHMI. Scientific foundations for future physicians. Retrieved August 18, 2010, from https://services.aamc.org/publications/showfile. cfm?file=version132.pdf\&prd_id=262\&prv_id=321\&pdf_id=132.

AMA. Results and implications of the AMA-APA physician mortality project. Stage II. Council on scientific affairs. JAMA. 1987;257 (21):2949-53.

Austin T R (2008) Pre-med education must be compatible with liberal arts ideals. Retrieved August 18, 2010 from http://www.insidehighered. com/views/2008/07/31/austin.

Barr DA, Gonzalez MA, et al. The leaky pipeline: factors associated with early decline in interest in premedical studies among underrepresented minority undergraduate students. Acad Med. 2008;83(5):503-11.

Beck AH. The Flexner report and the standardization of American medical education. JAMA. 2004;291(17):2139-40.

Center C, Davis C, et al. Confronting depression and suicide in physicians: a consensus statement. JAMA. 2003;289(23):3161-6.

Dalen JE, Alpert JS. Premed requirements: the time for change is long overdue! Am J Med. 2009;122(2):104-6. 
dePaula J (2009) Reforming pre-med. Retrieved August 18, 2010 from http://www.insidehighered.com/views/2009/08/20/depaula.

Dienstag JL. Relevance and rigor in premedical education. N Engl J Med. 2008;359(3):221-4.

Dobzhansky T. Biology, molecular and organismic. Am Zool. 1964;4:443-52.

Emanuel EJ. Changing premed requirements and the medical curriculum. JAMA. 2006;296(9):1128-31.

Flexner A. Medical education in the United States and Canada. A report to the Carnegie Foundation for the Advancement of Teaching. Bulletin No. 4. Boston: Updyke; 1910.

Gross JP, Mommaerts CD, et al. Perspective: after a century of criticizing premedical education, are we missing the point? Acad Med. 2008;83(5):516-20.

Harris. Physician shortage spreads across specialty lines. Retrieved November 15, 2010 from https://www.aamc.org/newsroom/reporter/ oct10/152090/physician_shortage_spreads_across_specialty_lines. html. 2010.

Kirch DG. Address to the AAMC annual meeting. Retrieved November 15, 2010, from https://www.aamc.org/newsroom/ newsreleases/2010/159528/101107.html. 2010.

Labov JB, Reid AH, et al. Integrated biology and undergraduate science education: a new biology education for the twenty-first century? CBE Life Sci Educ. 2010;9(1):10-6.

Muller D, Kase N. Challenging traditional premedical requirements as predictors of success in medical school: the Mount Sinai School of Medicine Humanities and Medicine Program. Acad Med. 2010;85(8):1378-83.

Nesse RM. Evolution: medicine's most basic science. Lancet. 2008;372:S21-7.

Nesse RM, Bergstrom CT, et al. Making evolutionary biology a basic science for medicine. Proc Natl Acad Sci USA. 2010;107 Suppl $1: 1800-7$.

O'Reilly. Physician shortage. Retrieved August 18, 2010, from http:// www.ama-assn.org/amednews/2010/03/29/prl20329.htm. 2010.

PMC. The case for personalized medicine. Retrieved August 18, 2010, from http://www.personalizedmedicinecoalition.org/about/ about-personalized-medicine/the-case-for-personalized-medicine. 2009.

Thomas L. Notes of a biology-watcher. How to fix the premedical curriculum. N Engl J Med. 1978;298(21):1180-1.

Ward L. Retrieved November 15, 2010, from https://www.aamc.org/ newsroom/newsreleases/2010/152932/101013.html. 2010.

West CP, Huschka MM, et al. Association of perceived medical errors with resident distress and empathy: a prospective longitudinal study. JAMA. 2006;296(9):1071-8.

Wilson DS. Evolution for everyone. How Darwin's theory can change the way we think about our lives. New York: Delacorte; 2007.

Wilson DS, Geher G, Waldo J. EvoS: completing the evolutionary synthesis in higher education. EvoS Journal: The Journal of the Evolutionary Studies Consortium. 2009;1(1):3-10. 